\title{
Dictionnaire de la philosophie russe, sous la direction de Mikhaïl Masline ; édition française sous la direction de Françoise Lesourd
}

Michel Niqueux

\section{(2) OpenEdition \\ Journals}

Édition électronique

URL : https://journals.openedition.org/cpuc/1072

DOI : $10.4000 /$ cpuc. 1072

ISSN : 2677-6529

Éditeur

Presses universitaires de Caen

\section{Édition imprimée}

Date de publication : 31 octobre 2011

Pagination : 239-249

ISBN : 978-2-84133-385-1

ISSN : $1282-6545$

Référence électronique

Michel Niqueux, "Dictionnaire de la philosophie russe, sous la direction de Mikhaill Masline ; édition française sous la direction de Françoise Lesourd », Cahiers de philosophie de l'université de Caen [En ligne], 48| 2011, mis en ligne le 01 septembre 2020, consulté le 07 février 2023. URL : http:// journals.openedition.org/cpuc/1072 ; DOI : https://doi.org/10.4000/cpuc.1072

\section{(@) $(1) \Theta$}

Creative Commons - Attribution - Pas d'Utilisation Commerciale 4.0 International - CC BY-NC 4.0

https://creativecommons.org/licenses/by-nc/4.0/ 


\section{Compte rendu}

Dictionnaire de la philosophie russe, sous la direction de Mikhaïl Masline [Moscou, Respublika, 1995; Moscou, Komplex, 2007], édition française sous la direction de Françoise Lesourd, Lausanne, l'Âge d'homme, 2010, 1016 p. ISBN 978-2-8251-4024-6

Ce monumental Dictionnaire est une traduction (plus ou moins adaptée) dirigée, et en partie réalisée par Françoise Lesourd (professeur à l'université de Lyon 3 , spécialiste de L. ${ }^{*}$ Karsavine ${ }^{1}$ ), de deux éditions d'un Dictionnaire de philosophie russe publié sous la direction de Mikhail Masline ${ }^{2}$. D'emblée se pose le problème du changement de destinataire: un dictionnaire conçu et écrit pour un public russe pouvait-il s'adresser sans grandes modifications à un public français? A priori, non, mais dans la mesure où le lecteur russe avait été privé pendant sept décennies de pans entiers de la pensée et de la philosophie de son pays (spiritualité de la Russie ancienne, slavophilisme, conservatisme, philosophie religieuse...), et que le dictionnaire source, l'un des premiers de l'époque post-soviétique, traitait précisément ces « taches blanches » comme des continents inconnus, la question du destinataire n'est pas cruciale. Pour rompre avec le schéma des Histoires de la philosophie publiées à l'époque soviétique, pour lesquelles toute la pensée russe tendait téléologiquement à l'apparition et à la domination du marxisme-léninisme 3 , les auteurs (sur lesquels on ne dispose pas de notices bio-bibliographiques) accordent une grande place à la préhistoire de la philosophie dans la Russie ancienne (XI ${ }^{\mathrm{e}}-\mathrm{XVII}{ }^{\mathrm{e}} \mathrm{s}$.). B. ${ }^{\star}$ Zenkovsky, au début de son Histoire de la philosophie russe, rappelait que «nous ne trouvons pas en Russie, avant le

1. L'astérisque indique qu'un article du Dictionnaire est consacré au nom ou au terme donné.

2. Professeur à l'université de Moscou, titulaire de la chaire d'histoire de la philosophie russe, né en 1947.

3. Il y a une entrée ${ }^{\star}$ Lénine, et une autre ${ }^{*}$ Marx en Russie, mais pas d'article sur le marxismeléninisme. 
XVIII ${ }^{e}$ siècle, d'ouvrages ou d'essais originaux de nature philosophique» 4 , mais cela, poursuivait-il, ne signifie pas que les Russes étaient auparavant étrangers à l'interrogation philosophique, celle-ci relevant d'une conception religieuse du monde et non d'une spéculation autonome. De fait, la spiritualité de la Russie ancienne influencera la philosophie religieuse de l'Âge d'argent (la renaissance artistique et intellectuelle de la fin du $\mathrm{XIX}^{\mathrm{e}}$ - début du XX $\mathrm{XX}^{\mathrm{e}}$ siècle, après une seconde moitié du XIX ${ }^{\mathrm{e}}$ siècle dominée par le ${ }^{\star}$ positivisme ${ }^{5}$ ), et méritait d'être traitée, bien qu'il ne s'agisse pas encore de philosophie. On trouvera ainsi des articles sur des saints et des figures de la spiritualité russe: ${ }^{\star}$ Sérapion de Vladimir, ${ }^{\star}$ Serge de Radonège, ${ }^{\star}$ Nil Sorki (de la Sora), chef de file des «non-possesseurs» (traduit ici par "adversaires de l'usage des biens du monde»), ${ }^{\star}$ Maxime le Grec; sur des phénomènes comme l`^ hésychasme, $l^{\text {**} a s c e ́ t i s m e, ~ l e ~ * ~ p a g a n i s m e, ~ o u ~ e n c o r e ~}$ le *synergisme et l’^icône.

Le Dictionnaire comprend trois types d'entrées: des personalia, des articles transversaux et des ouvrages.

Outre tous les grands philosophes et nombre d'auteurs peu ou pas connus (et les articles qui leur sont consacrés sont souvent sans équivalent en français), on trouve dans ce dictionnaire (listes non exhaustives):

- des théologiens et des prédicateurs: ${ }^{\star}$ Hilarion $\left(\mathrm{XI}^{\mathrm{e}} \mathrm{s}\right.$.); puis au XIX $\mathrm{X}^{\mathrm{e}}$ siècle, A. ${ }^{\star}$ Boukharev, ${ }^{\star}$ Ignati Briantchaninov, A. ${ }^{\star}$ Ivantsov-Platonov, F. ${ }^{\star}$ Goloubinski, V. ${ }^{\star}$ Koudriavtsev-Platonov, ${ }^{\star}$ Philarète; au XX ${ }^{e}$ siècle, ${ }^{\star}$ Jean de San-Francisco (mais pas Jean de Kronstadt), et d'autres, sur lesquels on trouvera plus de renseignements dans Les voies de la théologie russe, de G. ${ }^{\star}$ Florovski (ouvrage dont N. ${ }^{\star}$ Berdiaev critiqua radicalement l'antioccidentalisme dans ${ }^{*}$ Pout, $\left.\mathrm{n}^{\circ} 53,1937\right)$;

- des écrivains: N. ${ }^{\star}$ Gogol, A. ${ }^{*}$ Pouchkine, F. ${ }^{*}$ Dostoïevski, L. ${ }^{*}$ Tolstoï, mais il n'y a pas I. Tourgueniev ${ }^{6}$, ni M. Gorki, que l'énergétisme d'Ostwald, allié à l’ empiriomonisme de $\mathrm{A}$. ${ }^{\star}$ Bogdanov, et le nietzschéisme mâtiné de marxisme, conduira à la " ${ }^{*}$ construction de Dieu» et à la glorification de la «refonte» de l'homme par Staline;

- des poètes: V. ${ }^{\star}$ Joukovski, A. ${ }^{\star}$ Fet, F. ${ }^{*}$ Tiouttchev, A. ${ }^{\star}$ Blok, et un article sur la ${ }^{\star}$ poésie philosophique;

4. B. Zenkovsky, Histoire de la philosophie russe, Paris, Gallimard, t. I, 1953, p. 27.

5. Voir P. Pascal, Les grands courants de la pensée russe contemporaine, Lausanne, l'Âge d'homme, 1971 (rééd. 2010).

6. Voir H. Granjard, Ivan Tourguénev et les courants politiques et sociaux de son temps, Paris, Institut d'études slaves, 1966. 
- des historiens de la culture: I. ${ }^{\star}$ Lotman, P. ${ }^{*}$ Mouratov;

- des critiques littéraires: V. ${ }^{\star}$ Biélinski, N. ${ }^{\star}$ Nadiejdine;

- des musiciens: A. ${ }^{\star}$ Scriabine;

- des peintres: V. ${ }^{\star}$ Kandinski, K. ${ }^{*}$ Malévitch;

- des historiens: V. ${ }^{\star}$ Tatichtchev, N. ${ }^{\star}$ Karamzine, N. ${ }^{\star}$ Kostomarov, V. ${ }^{\star}$ Lamanski, S. ${ }^{\star}$ Soloviov (le père du philosophe), A. ${ }^{\star}$ Lappo-Danilevski, V. ${ }^{\star}$ Klioutchevski, P. ${ }^{\star}$ Milioukov;

- des personnages historiques: A. ${ }^{\star}$ Kourbski, contempteur du despotisme d'Ivan le Terrible; ${ }^{*}$ Catherine II; mais les Observations de Diderot sur le Nakaz (l'Instruction de l'Impératrice aux députés pour la confection des lois [1774]) ne sont pas citées;

- des hommes politiques: N. ${ }^{*}$ Boukharine, ${ }^{*}$ Lénine, L. ${ }^{\star}$ Trotski, mais pas J. Staline, sur lequel on trouvera une demi-page dans l'article Philosophie $\mathrm{du} *$ Pouvoir;

- des économistes: M. ${ }^{\star}$ Tougan-Baranovski, N. ${ }^{\star}$ Kondratiev;

- des linguistes: A. ${ }^{\star}$ Potebnia, R. ${ }^{\star} J a k o b s o n$, mais pas N. Marr ${ }^{7}$;

- des sociologues: M. ${ }^{\star}$ Kovalevski, L. ${ }^{\star}$ Metchnikov;

- des pychologues et des psychiatres: P. ${ }^{\star}$ Gannouchkine, A. ${ }^{\star}$ Léontiev, I. *Pavlov, I. ${ }^{\star}$ Sétchénov, M. ${ }^{\star}$ Troïtski, L. ${ }^{\star}$ Vygotski;

- des mathématiciens: ${ }^{\star}$ Lobatchevski.

Il y a quelques philosophes vivants (nés entre 1934 et 1941): A. *Akhoutine, N. ${ }^{\star}$ Avtonomova, $\mathrm{P} .{ }^{\star}$ Gaïdenko, S. ${ }^{\star}$ Khorouji, N. ${ }^{\star}$ Motrochilova (auteur, entre autres, d'un livre sur Husserl qui ne figure pas dans l'article ${ }^{\star}$ Husserl en Russie, ainsi que d'une grande étude sur la réception et l'édition de Kant en Russie ${ }^{8}$ ), V. ${ }^{\star}$ Stiopine, G. ${ }^{\star}$ Tchelpanov - G. ${ }^{\star}$ Gatchev étant décédé en 2008. Certains ont participé aux deux numéros de la revue Diogène consacrés à La philosophie en Russie: tendances et perspectives $\left(\mathrm{n}^{\circ} 222,2008 / 2\right.$ et $\left.\mathrm{n}^{\circ} 223,2008 / 3\right)$, qui ne sont pas mentionnés.

Le terme de "philosophie», est, on le voit, très extensif, et il aurait mieux valu que le Dictionnaire (ne serait-ce que pour en élargir le cercle des lecteurs) s'intitulât Dictionnaire de la pensée russe. Certes, on peut

7. Voir R. L'Hermitte, Marr, marrisme, marristes: une page de l'histoire de la linguistique soviétique, Paris, Institut d'études slaves, 1987.

8. Dans Kant-Studien, vol. 91, 2000, p. 73-95. 
arguer de l'absence de frontière nette en Russie entre les savoirs, et tous ces auteurs sont étudiés avant tout sous un angle philosophique - ce qui fait que le lecteur ignorera qu’A. *Biély, théoricien du *symbolisme, marqué par le néo-kantisme de $\mathrm{H}$. Cohen et de $\mathrm{H}$. Rickert (qui ne sont pas cités) puis de R. Steiner, est avant tout l'auteur de Pétersbourg (1916), l'un des grands romans du XX $\mathrm{XX}^{\mathrm{e}}$ siècle européen (traduit en français en 1967).

Les articles transversaux peuvent être groupés selon les rubriques suivantes :

- ${ }^{\star}$ Historiographie de la philosophie russe en Russie, et à l'étranger (où l'on s'étonne de ne pas voir citées les revues Studies in Soviet Thought, Studies in East European Thought, Russian studies in philosophy);

- lieux d'élaboration et de diffusion de la philosophie: *Académies de théologie (avec nombre d'articles consacrés à des professeurs de ces académies), monastère $d^{\prime}$ Optina poustyn, ${ }^{\star}$ Sociétés de philosophie et de philosophie religieuse, ${ }^{\star}$ Académie libre de culture philosophique et religieuse (1919-1923), ${ }^{\star}$ Institut de philosophie de l’Académie des sciences de Russie, ${ }^{\star}$ Enseignement de la philosophie en Russie, la philosophie dans les ${ }^{\star}$ universités, ${ }^{\star}$ revues de philosophie $\left({ }^{*}\right.$ Logos,${ }^{*}$ Voprosy filosofi i psikhologii, ${ }^{\star}$ Pout ayant droit à une entrée);

- domaines de la philosophie: ${ }^{\star}$ esthétique, ${ }^{\star}$ éthique, ${ }^{\star} \operatorname{logique},{ }^{*}$ ontologie (10 pages), philosophie de la ${ }^{\star}$ connaissance (l'un des articles les plus consistants, 11 pages), du *droit, de l’ histoire (l'article ne mentionne pas G. ${ }^{\star}$ Chpet, alors que $L^{\star}$ histoire comme problème de logique a droit à une entrée), du ${ }^{\star}$ pouvoir, des ${ }^{\star}$ sciences. La philosophie de l'érôs, qui est une «spécialité» de la philosophie religieuse russe (avec V. ${ }^{\star}$ Soloviov, B. ${ }^{\star}$ Vycheslavtsev, N. ${ }^{\star}$ Berdiaev) aurait mérité une entrée;

- courants philosophiques ou courants idéologiques: ${ }^{*}$ conservatisme, ${ }^{*}$ libéralisme, ${ }^{*}$ populisme, ${ }^{*}$ nihilisme, ${ }^{*}$ positivisme, ${ }^{*}$ théosophie, ${ }^{*}$ empiriocriticisme et ${ }^{\star}$ empiriomonisme, ${ }^{\star}$ intuitionnisme (avec N. ${ }^{\star}$ Losski et $\mathrm{S} .{ }^{\star}$ Frank), ${ }^{\star}$ existentialisme $\left(\mathrm{N} .{ }^{\star}\right.$ Berdiaev, principalement), ${ }^{\star}$ cher cheurs et ${ }^{\star}$ constructeurs de Dieu, ${ }^{\star}$ cosmisme, ${ }^{\star}$ eurasianisme, sur-représenté avec six auteurs: N. ${ }^{\star}$ Troubetskoï ${ }^{9}, \mathrm{P} .{ }^{\star}$ Savitski, G. ${ }^{\star}$ Florovski, P. ${ }^{\star}$ Souvtchinski, les «néo-eurasianistes » L. ${ }^{\star}$ Goumiliov, avec quatre pages de bibliographie (le record du Dictionnaire), et A. ${ }^{\star}$ Panarine;

9. Troubetskoï utilisait la transcription Troubetzkoy pour ses publications en français. Voir l'anthologie publiée par Patrick Sériot: N. Troubetzkoy, L^Europe et l'humanité, Liège, Mardaga, 1996, non mentionnée à l'article consacré à ce livre. 
ainsi que deux ouvrages, $L^{\star}$ Europe et l'humanité, et * Exode vers l'Orient et le concept de * personne symphonique. Il est vrai que cette idéologie imprègne fortement le discours dominant actuellement en Russie (conservatisme antimondialiste, antiuniversaliste, essentialisme culturel, avec le principal idéologue du néo-eurasisme, A. Douguine, absent du dictionnaire) mais encore aurait-il fallu l'indiquer ${ }^{10}$;

- histoire de la réception en Russie des philosophes étrangers: ${ }^{\star}$ Feuerbach, ${ }^{\star}$ Fichte, ${ }^{*}$ Hegel, ${ }^{\star}$ Husserl, ${ }^{\star}$ Kant $(8$ pages $),{ }^{\star}$ Marx,${ }^{*}$ Nietzsche,${ }^{*}$ Pascal, ${ }^{\star}$ Platon, ${ }^{\star}$ Schelling ${ }^{11},{ }^{\star}$ Schopenhauer, ${ }^{\star}$ Voltairianisme, ${ }^{\star}$ Wolffianisme. Mais comment se faire une idée de la réception des philosophes qui ne figurent pas dans cette liste, notamment Leibniz, Spinoza, Descartes, Rousseau, J. de Maistre, Herder ${ }^{12}$, Fourier ${ }^{13}$, Darwin ${ }^{14}$, Bergson ${ }^{15}$, Heidegger, etc. Un index des philosophes non russes s'avère indispensable;

- concepts philosophiques: concepts généraux, tels que *antinomisme, *néant, et concepts propres à un philosophe particulier: le *transrationnel et ${ }^{*}$ l'inconcevable pour $S$. ${ }^{*}$ Frank, suivi d'un article sur l'ouvrage du même nom, $\mathrm{l}^{\text {}}$ objectivation pour $\mathrm{N} .{ }^{*}$ Berdiaev, le ${ }^{\star}$ déracinement et la ${ }^{\star}$ lutte pour l'impossible pour L. ${ }^{\star}$ Chestov, etc. Les concepts de la philosophie religieuse forment un riche ensemble: ${ }^{\star}$ anthropothéisme $v{ }^{*}$ *divino-humanité, ${ }^{*}$ métaphysique du cœur, *intégrité, ${ }^{*}$ sobornost (conciliarité), ${ }^{\star}$ unitotalité (8 pages), ${ }^{\star}$ sophiologie (8 pages), philosophie $\mathrm{du}{ }^{\star}$ nom, ${ }^{\star}$ onomatodoxes, ${ }^{\star}$ ascétisme. Là aussi, un index des concepts aurait permis de suivre leur migration et leur évolution;

- articles d'histoire des idées: ${ }^{\star}$ narodnost (esprit national) ${ }^{16},{ }^{*}$ commune paysanne, ${ }^{*}$ coopération, ${ }^{*}$ Moscou troisième Rome, ${ }^{\star}$ Orient et Occi$\operatorname{dent}^{17}$, dissidence religieuse $\left({ }^{\star}\right.$ double foi, une notion à discuter, le peuple

10. Sur ce sujet, voir M. Laruelle, La quête d’une identité impériale. Le néo-eurasisme dans la Russie contemporaine, Paris, Petra, 2007.

11. Bibliographie limitée à deux titres, sans l'ouvrage fondamental dirigé par В. Ф. Пустарнаков, Философия Шеллинга в России, СПб., Изд-во РХГИ, 1998.

12. Voir J. Zeldin, "Herder and Some Russians", in Western Philosophical Systems in Russian Literature. A Collection of Critical Studies, A. M. Mlikotin (dir.), Los Angeles, University of Southern California Press, 1979, p. 11-24.

13. Voir G. Sourine, Le fouriérisme en Russie, Paris, Dupont, 1936.

14. Sur le darwinisme en Russie, voir l'étude de V. Barkov in Dictionnaire du darwinisme et de l'évolution, P. Tort (dir.), Paris, PUF, 1996, t. 1, p. 1044-1108.

15. Voir F. Nethercott, Une rencontre philosophique: Bergson en Russie (1907-1917), Paris, L'Harmattan, 1995.

16. Voir l'excellente mise au point de A. Miller, «Natsiia, Narod, Narodnost' in Russia in the $19^{\text {th }}$ Century: Some Introductory Remarks to the History of Concepts ", Jahrbücher für Geschichte Osteuropas, vol. 56, n 3, 2008, p. 379-39o.

17. Voir M. Dennes, Russie-Occident, philosophie d'une différence, Paris, Mentha, 1991. 
pratiquant une religion populaire dans laquelle seul le chercheur distingue les survivances païennes des éléments chrétiens, ${ }^{\star}$ vieux-croyants, *judaïsants), mouvements politiques: ${ }^{\star}$ décembristes, ${ }^{\star}$ panslavisme, *Jeunes Russes (monarchistes de l'émigration);

- histoire de l'art: ${ }^{\star}$ symbolisme, ${ }^{\star}$ futurisme, ${ }^{*}$ conceptualisme, mais rien sur le réalisme socialiste.

Pour les ouvrages (moins d'une trentaine), les philosophes de l'Âge d'argent se taillent la part du lion: G. ${ }^{\star}$ Chpet et $S$. ${ }^{*}$ Frank ont trois de leurs ouvrages qui sont analysés, V. ${ }^{\star}$ Soloviov et $\mathrm{S}$. ${ }^{\star}$ Boulgakov - deux, et un pour P. ${ }^{\star}$ Florenski, N. ${ }^{\star}$ Berdiaev, N. ${ }^{\star}$ Losski, L. ${ }^{\star}$ Chestov, A. ${ }^{\star}$ Lossev. Auteur et ouvrage ont souvent des rédacteurs différents, ce qui entraîne des redondances.

Circuler dans ce gros dictionnaire n'est pas facile pour celui qui veut s'initier à la philosophie ou à la pensée russe: l'unique index consiste en une liste alphabétique des entrées du dictionnaire, sans dates pour situer les auteurs, et sans le nom de l'auteur des ouvrages étudiés. Un index thématico-chronologique aurait permis de regrouper les entrées (au nombre de plus de 400) en grands ensembles - Russie ancienne, XVIII siècle, première et seconde moitié du XIX ${ }^{\mathrm{e}}$ siècle (respectivement placés sous le signe de l'idéalisme allemand et du positivisme), philosophie religieuse de l'Âge d'argent (qui se poursuit dans l'émigration), philosophie soviétique, etc. -, et aurait offert au lecteur des tableaux de ce qui est proposé. Ainsi, pour le XVIII ${ }^{\mathrm{e}}$ siècle (choisi pour exemple comme étant le plus bref), outre certains des articles transversaux cités plus haut, dont *voltairianisme et ${ }^{*}$ wolffianisme, ainsi que celui sur les ${ }^{\star}$ Lumières, on verrait se dessiner:

- un groupe d'articles sur la pensée religieuse: *Théologie philosophique des XVIII ${ }^{\mathrm{e}}-\mathrm{XX}^{\mathrm{e}}$ siècle; ${ }^{\star}$ franc-maçonnerie ${ }^{18}$, à laquelle se rattache N. ${ }^{\star}$ Novikov; les frères ${ }^{\star}$ Likhoude, ${ }^{\star}$ Païssi Vélitchkovski et la ${ }^{\star}$ Philocalie (traduite en français, comme ne l'indique pas l'article), ${ }^{\star}$ Séraphin de Sarov, ${ }^{*}$ Féofan Prokopovitch, les ${ }^{\star}$ Réponses $d u$ Pomorié des ${ }^{\star}$ vieuxcroyants, et surtout la figure du premier philosophe russe (ukrainien), G. *Skovoroda (c'est par lui qu'Alexandre Papadopoulo commence son Introduction à la philosophie russe [Paris, Seuil, 1995]), pour lequel il aurait fallu indiquer qu'il est l'auteur de la «Profession de foi » des

18. Voir Slavica occitania [Toulouse], $\mathrm{n}^{\circ}$ 24, 2007, La franc-maçonnerie et la culture russe, J. Breuillard, I. Ivanova (dir.). 
doukhobors, ces «chrétiens spirituels » que L. *Tolstoï aidera à émigrer en 1898 au Canada ${ }^{19}$;

- un groupe d'écrivains: A. ${ }^{\star}$ Bolotov, F. ${ }^{\star}$ Émine, D. ${ }^{\star}$ Fonvizine, A. ${ }^{\star}$ Kantémir, N. ${ }^{\star}$ Karamzine et le ${ }^{\star}$ sentimentalisme, M. ${ }^{\star}$ Lomonossov, bien étudiés par ailleurs dans le premier volume de l'Histoire de la littérature russe de chez Fayard, Des origines aux Lumières (Paris, 1992);

- la philosophie du pouvoir, avec ${ }^{\star}$ Catherine II, A. ${ }^{*}$ Radichtchev, et l'historien M. ${ }^{\star}$ Chtcherbatov.

D'autres tableaux synoptiques permettraient de voir d'emblée par qui est représentée la philosophie soviétique ou celle de l'émigration, ou encore de lier un nom, par exemple $S$. ${ }^{\star}$ Frank, à un ouvrage ( $L^{\star *}$ objet du savoir) et à un concept (l’^Inconcevable), soit trois articles qu'il est impossible de réunir d'après l'index, et même à la lecture de l'article sur ${ }^{\star} F r a n k$, puisqu'il n’y a pas de renvoi à l’^Inconcevable dans cet article...

Le contenu de tous ces articles ne peut être discuté ici, et du reste il n'appelle pas de réserves particulières. Les articles, de une à dix pages, sont denses (d'autant plus qu'il n'y a pas le moindre paragraphe), généralement bien construits. Les problèmes de traduction terminologique ont été bien résolus: F. Lesourd s'en explique dans sa Présentation, et cite comme référence le Vocabulaire européen des philosophies. Dictionnaire des intraduisibles (B. Cassin [dir.], Paris, Le Robert - Seuil, 2004), mais sans que les articles comme ${ }^{*}$ sobornost, mir ( ${ }^{*}$ commune rurale) l'utilisent; la transcription et la translittération sont bien employées. Il n'y a pas d'erreurs factuelles ${ }^{20}$, les maladresses d'expression sont rares: ${ }^{\star}$ Hilarion $\left(\mathrm{XI}^{\mathrm{e}} \mathrm{s}\right.$. $)$ - «idéologue du christianisme russe», le «mysticisme obscurantiste» du prince A. Golitsyne (p. 928). Ce sont plutôt des éclairages différents ou des compléments que l'on pourrait souhaiter: une approche plus critique, au sens scientifique du terme, plus comparatiste, moins russo-centriste (on ne trouvera rien, par exemple, sur N. ${ }^{\star}$ Berdiaev et $\mathrm{E}$. Mounier, alors que l'influence du premier

19. Une thèse a été soutenue en 1994 à l'université Paris-Sorbonne (Paris-IV) par E. Volsky sur La pensée de G. Skovoroda et ses sources antiques. Voir aussi Skovoroda, philosophe ukrainien, Paris, Institut d'études slaves, 1976.

20. Toutefois, le "père» du syntagme et du concept d' "`Idée russe» n'est pas V. *Soloviov (dans un livre initialement publié sous ce titre en français à Paris en 1888 ; réédité dans La Sophia et les autres écrits en français, Paris, l'Âge d'homme, 1978), mais Dostoïevski, dans le prospectus de la revue Vremia, en 1861. L'«idée russe» désigne la "voie russe», messianique, du développement de la Russie, qui du reste lui a souvent été suggérée par des penseurs occidentaux (Voltaire, Baader, Schelling, Tocqueville, etc.). Voir A. Bourmeyster, L'idée russe entre Lumières et spiritualité sous le règne de Nicolas $I^{e r}$, Grenoble, Ellug, 2001. 
sur les débuts d'Esprit et du personnalisme est avérée ${ }^{21}$ ), des indications sur la réception des œuvres, les polémiques qu'elles sont suscitées. Une meilleure articulation des articles entre eux aurait aussi été souhaitable: il manque des renvois (un seul exemple: dans l'article sur *Platon en Russie, on ne trouve pas les noms de N. ${ }^{\star}$ Nadiejdine, qui publia en 1830 une série d'articles sur Platon, ni celui de A. ${ }^{\star}$ Blok, à qui «les idées platoniciennes donnèrent l'impulsion première à sa création poétique» [p. 94], ni celui de P. *Iourkiévitch). Les articles semblent s’ignorer les uns les autres. Bien que des synthèses aient préalablement été effectuées à partir des articles du dictionnaire source, d'autres auraient pu être réalisées avec les articles retenus. Quand on a, pour un auteur donné, un article à son nom, un autre sur une de ses œuvres, et un troisième sur un de ses concepts, les répétitions sont inévitables, surtout lorsque les plumes sont différentes. Il en va de même pour les articles qui portent sur des thèmes proches: ${ }^{*}$ enseignement de la philosophie en Russie et philosophie dans les ${ }^{\star}$ universités, philosophie du ${ }^{\star}$ nom et ${ }^{\star}$ onomatodoxes, N. ${ }^{*}$ Fiodorov et ${ }^{*}$ psychocratie, N. ${ }^{\star}$ Danilevski et les ${ }^{\star}$ types historico-culturels, etc. La plupart des compléments donnés dans le «Lexique» final (une trentaine de pages) auraient pu facilement être intégrés aux articles. Supprimer les répétitions, fondre plusieurs articles apparentés en un seul aurait libéré de la place pour des index et des alinéas.

Chaque article est suivi d'une bibliographie plus ou moins importante, divisée en Euvres et Études. Dans la mesure où certaines comportent, outre des références russes, les traductions françaises des œuvres et des études en français (et parfois en anglais), le lecteur est en droit de considérer que l'absence de telles références signifie qu'il n'y a rien en français sur le sujet. Qu'il n'en croie rien! Innombrables sont les références françaises «incontournables» qui manquent, tant pour les œuvres que pour les études, et que les moteurs de recherche ou les catalogues informatisés ne trouveront pas toujours: il en va ainsi des textes de la Russie ancienne cités dans le Dictionnaire, et qui sont traduits (au moins en extraits) dans le Patrimoine littéraire européen (Paris, De Boeck université, 14 volumes parus de 1992 à 2000). On trouvera dans le volume 4a (1993) le Sermon sur la foi et la grâce d’^Hilarion, des extraits du ${ }^{\star}$ Dit de l'ost d'Igor, dont il existe au moins sept traductions françaises, sans qu'aucune ne soit citée, des sermons de *Sérapion; le volume 9 (Les Lumières, de l'Occident à l'Orient, 1997) renferme des traductions d'auteurs du XVIII ${ }^{\mathrm{e}}$ siècle (A. ${ }^{\star}$ Bolotov, F. Émine, M. ${ }^{\star}$ Chtcherbatov, N. ${ }^{\star}$ Novikov, D. ${ }^{\star}$ Fonvizine, M. ${ }^{\star}$ Lomonossov) ainsi que du Domoströ̈ ${ }^{\star}$ Ménagier) du $\mathrm{XVI}^{\mathrm{e}}$ siècle,

21. Voir O. Clément, Berdiaev. Un philosophe russe en France, Paris, Desclée de Brouwer, 1991. 
par ailleurs intégralement traduit en 1910 par E. Duchesne (Paris, Picard). Comment deviner, aussi, que l'on peut trouver le texte français du Règlement ecclésiastique de ${ }^{\star}$ Féofan Prokopovitch au nom de Pierre le Grand ${ }^{22}$, que l'Histoire du règne de Jean IV (Ivan le Terrible) du prince *Kourbski est éditée en français, de même que les lettres d'Ivan le Terrible à celui$\mathrm{Ci}^{23}$, etc. On trouvera aussi un choix de textes inédits de philosophes du XVIII ${ }^{\mathrm{e}}$ siècle dans La pensée des Lumières en Russie ${ }^{24}$. Pour le XIX ${ }^{\mathrm{e}}$ siècle, les traductions de référence (avec préface, notes et commentaires) ignorées des bibliographies sont, entre autres, les CEuvres complètes de N. ${ }^{*} \mathrm{Gogol}^{25}$, avec les Passages choisis de ma correspondance avec mes amis analysés dans l'article du Dictionnaire; les Essais philosophiques de I. ${ }^{\star}$ Kiréievski; Les nuits russes du schelligien $\mathrm{V} .{ }^{*}$ Odoïevski; les Mémoires d'outre-tombe de V. ${ }^{*}$ Pétchérine ${ }^{26}$, le Que faire? de N. ${ }^{*}$ Tchernychevski (Paris, Éditions des Syrtes, 2000); le texte original en français du mémorandum de $S .{ }^{*}$ Ouvarov dans lequel apparaît pour la première fois (1832) la fameuse triade du «nationalisme officiel» sous la forme «Religion nationale, Autocratie, Nationalité [ ${ }^{\star}$ Narodnost] ${ }^{27}$, etc. Même l'Histoire de la philosophie russe des origines à 1950, de N. ${ }^{\star}$ Losski (Paris, Payot, 1954) n’est donnée que dans la version anglaise. V. ${ }^{\star}$ Bibikhine figure dans le recueil $L$ 'unité ${ }^{28}$, avec S. ${ }^{*}$ Khorouji et d'autres. Au XIX ${ }^{\mathrm{e}}$ siècle, nombre de penseurs russes ont écrit directement en français; les éditions de ces textes auraient dû être indiquées: la Correspondance (1838-1842) entre I. Gagarine (converti au catholicisme) et G. ${ }^{\star}$ Samarine (Paris, Plamia, 2002), les articles politiques de F. ${ }^{\star}$ Tiouttchev (Paris, YMCA-Press, 1976), plusieurs écrits importants (en particulier la Lettre à Michelet) de A. ${ }^{\star}$ Herzen.

Aux Éditions en langues étrangères de Moscou ont paru à l'époque soviétique de bonnes anthologies de V. ${ }^{*}$ Biélinski, A. ${ }^{\star}$ Herzen, P. ${ }^{\star}$ Tkatchov, D. ${ }^{\star}$ Pissarev, ce dernier (idéologue du ${ }^{\star}$ nihilisme) figurant aussi, avec N. ${ }^{*}$ Tchernychevski et N. Dobrolioubov dans Les nihilistes russes. Textes choisis (Paris, Aubier-Montaigne, 1974) de Wanda Bannour, auteur

22. Règlement ecclésiastique, composé... par ordre de Pierre I ${ }^{e r}$, empereur de toutes les Russies, Paris, 1874.

23. Prince A. Kourbski, Histoire du règne de Jean IV (Ivan le Terrible), Genève, Droz, 1965 (préface et trad. par M. Forstetter); Ivan le Terrible, Correspondance avec le prince Kourbski. Épitres, Paris, Seghers, 1959 (trad. par D. Olivier).

24. La pensée des Lumières en Russie, opuscules choisis et traduits par le Groupe de travail du Centre de recherches de philosophie russe et soviétique de l'université de Lille III, B. Jeu (dir.), Lille, université de Lille III et Paris, Éditions universitaires, 1973.

25. N. Gogol, Euvres complètes, Paris, Gallimard (Bibliothèque de la Pléiade), 1975.

26. Dans Spicilegium historicum Congregationis sanctissimi Redemptoris, 52, 2, 2004, p. 255-355.

27. Mémorandum édité par A. Zorin dans Novoe literaturnoe obozrenie, 26, 1997, p. 92-95.

28. Société Vladimir Soloviev, L’unité, Fribourg, Éditions universitaires de Fribourg, 1996. 
également d'une étude sur Les nihilistes russes (Paris, Anthropos, 1978). Pour le * populisme, on est frappé par l'absence de la somme de F. Venturi, Les intellectuels, le peuple et la révolution. Histoire du populisme russe au $X I X^{e}$ siècle (Paris, Gallimard, 1972, 2 vol.), et il aurait été bon de rappeler les origines du terme ${ }^{29}$. L'ouvrage d'E. Lavigne, Introduction à l'histoire du nihilisme russe (Paris, G. Charpentier, 1880) reste précieux. C. *Pobédonostsev, M. ${ }^{\star}$ Kovalevski, L. ${ }^{\star}$ Metchnikov, L. ${ }^{\star}$ Tikhomirov, M. ${ }^{\star}$ Tougan-Baranovski et d'autres auteurs peu connus du début du $\mathrm{XX}^{\mathrm{e}}$ siècle ont été traduits en français de leur temps.

Les références aux études en français sont tout aussi lacunaires, donnant l'impression que la recherche française sur la philosophie russe est pratiquement inexistante.

Sur N. * Fiodorov, les articles de J. Grunwald dans Contacts. Revue française de l'orthodoxie ( $\left.\mathrm{n}^{\circ} 58,1967 ; \mathrm{n}^{\circ} 61,62-63,1968\right)$ sont indispensables et l'on traduit mieux voskrechenie par le substantif verbal actif ressuscitation, à la suite de C. Andronikof, traducteur de l'Histoire de la philosophie russe de B. *Zenkovsky, que par ${ }^{\star}$ résurrection. Il y a quelques références aux travaux de Jutta Scherrer, mais ses articles sur D. ${ }^{\star}$ Mérejkovski ${ }^{30}$, sur les ${ }^{\star}$ chercheurs de Dieu et les ${ }^{\star}$ constructeurs de Dieu ${ }^{31}$ manquent. Sur G. ${ }^{\star}$ Chpet et A. ${ }^{\star}$ Lossev, ont paru deux monumentaux recueils d'articles, dans deux livraisons de Slavica occitania $\left(\mathrm{n}^{\circ} 26,2008\right.$ et $\left.\mathrm{n}^{\circ} 31,2010\right)$. Il y aurait encore à citer des études sur F. ${ }^{\star}$ Émine (R. Baudin), ${ }^{\star}$ Moscou-Troisième Rome (L. Poliakov), la philosophie $d u^{\star}$ nom (A. Nivière, M. Dennes, F. Corrado), A. ${ }^{\star}$ Potebnia (J. Fontaine), C. ${ }^{\star}$ Pobédonostsev ${ }^{32}, \mathrm{~V} .{ }^{\star}$ Ern (J. Scherrer), N. ${ }^{\star}$ Boukharine (S. Cohen), ${ }^{\star}$ Lénine (D. Colas, A. Besançon), N. ${ }^{\star}$ Roerich (D. Savelli), sur la res rhetorica russe de M. ${ }^{\star}$ Spéranski (R. Gorbounova). Pour la philosophie soviétique, le lecteur doit être renvoyé aux ouvrages de R. Zapata ${ }^{33}$, auteur par ailleurs d'un «Que sais-je?» sur La philosophie russe et soviétique (1988),

29. Voir R. Pipes, «Narodnichestvo, a semantic inquiry», Slavic review, XXIII, 3, 1964, p. 441-458.

30. J. Scherrer, «Pour une théologie de la révolution. Merejkovski et le symbolisme russe», Archives des sciences sociales des religions, 45/1, 1978, p. 27-50.

31. J. Scherrer, "L’intelligentsia russe: sa quête de la "vérité religieuse du socialisme" ", Le temps de la réflexion, II, 1981, p. 113-152.

32. J. Salem, «Critiques de la démocratie parlementaire dans la Russie de la fin du XIX ${ }^{\mathrm{e}}$ siècle: Constantin Pétrovitch Pobedonostsev, théoriciens de l'autocratie ", Revue de métaphysique et de morale, $\mathrm{n}^{\circ} 45,2005 / 1$, p. 127-149.

33. R. Zapata, Luttes philosophiques en URSS 1922-1931, Paris, PUF, 1983, avec des textes de ${ }^{\star}$ Déborine, Minine, Stépanov et un chapitre sur le débat entre « ${ }^{\star}$ Dialecticiens » et «Mécanicistes». 
et de B. Jeu ${ }^{34}$. ${ }^{\star}$ Hegel en Russie a été étudié par A. Koyré ${ }^{35}$. Sur ${ }^{\star}$ Nietzsche en Russie, la bibliographie ne donne rien en français ${ }^{36}$. La pensée politique des décembristes a fait l'objet d'une importante thèse ${ }^{37}$.

La bibliographie sur F. ${ }^{*}$ Dostoïevski est importante, mais deux ouvrages spécialement consacrés à sa philosophie manquent: de L. A. Zander ${ }^{38}$, et de S. ${ }^{\star}$ Hessen ${ }^{39}$.

On ne trouve pratiquement aucune référence aux nombreux articles publiés dans Russian Thought, ni aux volumes trilingues (russe, polonais, anglais) publiés sous la direction de A. de Lazari ${ }^{40}$.

Ce ne sont là que quelques références que le lecteur est en droit d'attendre d'un outil de travail, qui doit précisément procéder à un tri et à un choix de références.

Pour conclure ce trop lapidaire compte rendu, tout en admirant le considérable travail de traduction et d'adaptation qui a été fait à partir des dictionnaires source, on ne peut que déplorer les nombreuses lacunes ou imperfections, qui rendent difficile l'exploitation de toutes les richesses amassées dans ce Dictionnaire. Mais que le lecteur y pénètre courageusement: il serait dommage de ne l'utiliser que pour des renseignements ponctuels, sans tenter de s'y frayer des parcours de recherche.

Michel Niqueux

Université de Caen Basse-Normandie

34. B. Jeu, La philosophie soviétique et l'Occident. Essai sur les tendances et la signification de la philosophie soviétique contemporaine (1959-1969), Paris, Mercure de France, 1969. B. Jeu est l'auteur du chapitre sur la philosophie russe dans l'Histoire de la philosophie de la Pléiade (Paris, Gallimard, 1974, t. 3, p. 856-901).

35. A. Koyré, Études sur l'histoire de la pensée philosophique en Russie, Paris, J. Vrin, 1950, p. $103-170$.

36. Voir M. Aucouturier, «Les trois Nietzsche russes", in Le rayonnement européen de Nietzsche, G. Merlio, P. D’Iorio (dir.), Paris, Klincksieck, 2004, p. 89-99; M. Niqueux, «Qu'en est-il de l'influence de Nietzsche sur Gorki? ", Slavica occitania [Toulouse], nº 22, 2006, p. 401-426.

37. J. Morel-Grandhaye, La République des décembristes. Pour une histoire de la modernité politique en Russie (1760-1870), M.-P. Rey (dir.), université Paris 1 Panthéon-Sorbonne, 2008, 3 vol. (dactyl.).

38. L. A. Zander, Dostoïevsky. Le problème du Bien, Paris, Corrêa, 1946.

39. S. Hessen, «La lutte entre l'utopie et l'autonomie du bien dans la philosophie de Dostoïevski et de Vl. Soloviev», Le Monde slave, 1930: $\mathrm{n}^{\circ}$ 2, p. 224-250; $\mathrm{n}^{\circ}$ 4, p. 41-58; n 7, p. 18-38; $\mathrm{n}^{\circ}$ 8, p. 186-201.

40. Idei v Rossii. Idee w Rosji. Ideas in Russia. Leksykon rosyjsko-polsko-angielski, A. de Lazari (dir.), t. 1-5, Varsovie, Semper, 1999-2003. 